\title{
SYNTAX and Coronary Artery Calcium Score Predict Atherosclerotic Plaque Formation in the Ascending Aorta
}

\author{
Ismail Haberal, ${ }^{1}$ Mehmet Balli, ${ }^{2}$ Esra Erturk Tekin, ${ }^{3}$ Ayhan Uysal, ${ }^{4}$ Sadiye Deniz Ozsoy, ${ }_{1}$ Mehmet Ali Yesiltas, ${ }^{5}$ \\ Dogaç Oksen, ${ }^{6}$ Sebnem Batur, ${ }^{7}$ Hulya Yilmaz Ak, ${ }^{8}$ Bahar Aydinli ${ }^{9}$ \\ ${ }^{1}$ Department of Cardiovascular Surgery, İstanbul University - Cerrahpasa, Institute of Cardiology, İstanbul, Turkey; ${ }^{2}$ Department \\ of Cardiology, Mersin City Training and Research Hospital, Mersin, Turkey; ${ }^{3}$ Department of Cardiovascular Surgery, Mersin City \\ Training and Research Hospital, Mersin, Turkey; ${ }^{4}$ Department of Cardiovascular Surgery, Firat University Medical Faculty, Elazig, \\ Turkey; ${ }^{5}$ Department of Cardiovascular Surgery, Health Ministry Bakırköy Dr. Sadi Konuk Training and Research Hospital, İstanbul, \\ Turkey; ${ }^{6}$ Department of Cardiology, İstanbul University - Cerrahpasa, Institute of Cardiology, İstanbul, Turkey; ${ }^{7}$ Department of \\ Pathology, İstanbul University, İstanbul University Cerrahpaşa Medical Faculty, İstanbul, Turkey; ${ }^{8}$ Department of Anesthesiology \\ and Reanimation, İstanbul University - Cerrahpasa, Institute of Cardiology, İstanbul, Turkey; ${ }^{9}$ Department of Anesthesiology and \\ Reanimation, Mersin City Training and Research Hospital, Mersin, Turkey
}

\section{ABSTRACT}

Background: There is no study about the relationship between the complexity of coronary artery disease (SYNTAX SCORE; SS), and coronary artery calcium (CAC) score, accompanied with aortic calcium score (ACS) levels. The objective of this study was to investigate the relationship between the preoperative SS and CAC scores accompanying ACS in isolated CABG patients and their postoperative clinical results.

Methods: This study included 130 consecutive CABG patients. The mean age of the patients was $62.3 \pm 8.62$ years (range: 47-84 years). SS was measured using coronary angiography by an experienced cardiologist. We investigated the ACS accompanied with CAC scores using a multidetector computed tomography (MDCT) in the same session, preoperatively. Measurements of the CAC score and ACS were measured by an experienced radiologist, who was unaware of the study in the same session. In order to investigate aortic wall pathology in patients with positive aortic calcification, we provided aortic tissue samples prior to the proximal anastomosis of bypass grafts using No:11 scalpel.

Results: Overall median SS was $39 \pm 7.2$ (range: 15-41). CAC score was zero in 34 patients $(26.1 \%)$. For the patients with a CAC score of zero, the median SS was $32 \pm 9.4$. There was no evidence of aortic calcification or plaque formation in 62 patients (47.6\%). In these patients, the median SS was 35.6 \pm 11 . No significant difference was found when both groups were compared and for those patients with a calcific score of zero $(P=.85)$. The median CAC score and ACS were $238 \pm$ 122 AU (range: 0-1238 AU) and $112 \pm 40$ AU (range: 0-730 AU), respectively $(P=.0033)$. For patients with a CAC score

Received August 15, 2021; received in revised form September 7, 2021; accepted September 7, 2021.

Correspondence: Dr. Ismail Haberal, Istanbul University Institute of Cardiology, Department of Cardiovascular Surgery, Istanbul, Turkey, GSM; +905056239874 (e-mail:drismailhaberal@gmail.com). and ACS $\geq 400$ AU, the mean SYNTAX score was $\geq 37$. SS was correlated with CAC score (R:0.585; $P<.0001)$. SYNTAX was correlated with ACS (R:0.557; $P<.001)$. In multivariate analysis of SS (OR 1.053, 95\% CI: $1.003-1.106, P=.039)$, gender (OR 0.189, 95\% CI: 0.053-0.678, $P=0.011$ ), age (OR 1.454, 95\% CI: $1.256-1.632, P=.012)$, and diabetes mellitus (OR 0.341, 95\% CI: 1.006-1.124, $P=.014$ ) were independent predictors for CAC score and aortic calcification.

Conclusions: CAC score and ACS are strongly correlated with the complexity of coronary arteries in CABG patients. The total CAC score ( $\geq 400 \mathrm{AU}$ ) was independently associated with the degree of SS (>37). To prevent MACCE and mortality in CABG patients, we suggest the measurement of CAC score accompanied with ACS using MDCT as a noninvasive method.

Highlight points:

- Atherosclerotic plaque formation in aorta and coronary arteries are the main risk factors for stroke and infarction in CABG operations.

- SYNTAX score value and aortic atherosclerosis levels are directly correlated.

- $\quad$ SYNTAX score may predict the complications due to atherosclerosis during heart surgery.

\section{INTRODUCTION}

Traditional coronary angiography is accepted as the gold standard for the diagnosis of CAD, which may be used as a guide for suitable treatment options in patients with a severe CAD [Herrington 2016]. Previous reports demonstrated that the severity and complexity of CAD assessed by the use of SS-I or SS-II are associated with a high rate of major adverse effects of cerebral and cardiovascular events (MACCE) and perioperative myocardial infarction [Schaftenaar 2016; Kojima 2019; Nohara 2004]. SS was described as the prediction of the complexity of coronary artery lesions, and it was assigned using angiographic visualization. According to 


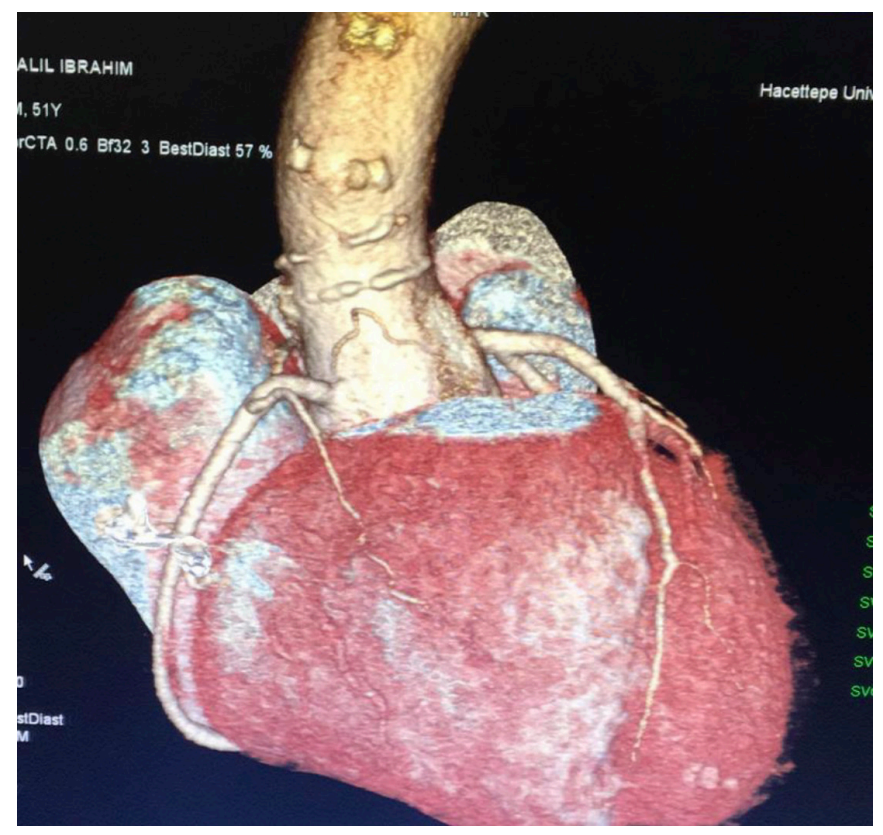

Figure 1. Aortic stiffness was measured from the sinotubular junction to the end of the aortic arch.

previous reports, patients with high SS were correlated with contrast nephropathy [Ozturk 2016] and new onset of atrial fibrillation [Rencuzogullari 2018]. Therefore, the evaluation of SYNTAX may prevent mortality and morbidity in patients with risk factors [Raggi 2000; Haberl 2001]. Coronary artery calcific disease and the aortic calcification score are other independent risk factors for mortality and morbidity, such as MACCE [Ozturk 2016; Rencuzogullari 2018; Raggi 2000]. However, the relationship between SS and CAC score and aortic stiffness has been reported in a limited number of case series [Budoff 2002; Shimizu 2015].

Properties of plaques and calcium load are important factors for vascular dissection or embolic events during stent placement in PCI procedures and aortic cross-clamping in CABG surgery. The processes, such as the presence of calcified plaque, remodeling of endothelial plaque, intracoronary or intra-aortic plaque rupture, and an endothelial dysfunction related to calcific disease, were described as the criteria for plaque formation [Raggi 2000; Haberl 2001; Budoff 2002; Shimizu 2015]. According to the major and minor criteria, MDCT may be used for assessing the risk of plaque vulnerability [Haberl 2001; Budoff 2002; Shimizu 2015]. Active inflammation, intravascular thrombosis, fibrous atherosclerosis with large lipid cells, and severe luminal stenosis are the major factors for plaque vulnerability.

Frequently, angiographically proven CAD is associated with coronary calcification. Because coronary calcification is an independent predictor of cardiovascular events, MDCT is proposed for the detection of plaque formation, including intravascular or extravascular calcific load [Ruben 2018; Mirbolouk 2020]. Atherosclerotic plaque rupture may cause an aortic aneurysm or dissection, penetrating ulcer, and cerebral embolic events. Therefore, cardiac MDCT is being increasingly used

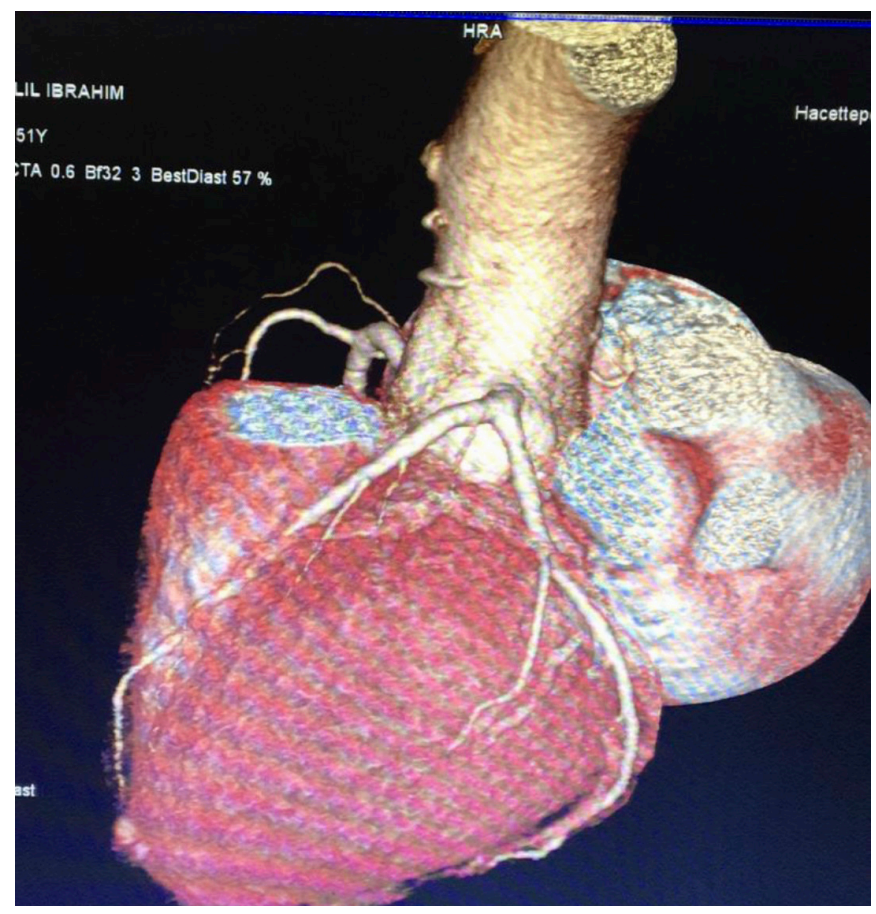

Figure 2. Sinotubular junction to end of the aortic arch to calculate calcific score.

as a noninvasive imaging method in clinical settings for screening in the suspicion of vascular calcific disorders [Raggi 2000; Haberl 2001; Budoff 2002; Shimizu 2015].

The authors showed that the CAC score and/or aortic calcification [Raggi 2000; Haberl 2001; Budoff 2002] were independent predictors of mortality and morbidity in CABG or PCI patients. Shimizu et al demonstrated that aortic valve calcification could be a surrogate marker for CAD progression [Shimizu 2015]. They suggested the routine ACS measurement from an aortic annulus to descending aorta to prevent the post-procedure risk of mortality in patients with severe aortic valvular calcification. Ruben et al demonstrated that abdominal aortic calcification was associated with an increased burden of cardiovascular morbidity and mortality, and it could be used as a predictor of adverse cardiovascular disease events [Ruben 2018]. Mirbolouk et al showed that a high CAC score was an independent risk factor of all-cause mortality and related to cardiovascular events [Mirbolouk 2020]. Calcific scoring is a non-invasive imaging method with relatively little radiation exposure.

Our aim in this study was to investigate the relationship between the coronary artery and aortic calcium scoring and SS to investigate whether these measurements can be used as predictors to prevent cardiovascular mortality and morbidity in $\mathrm{CABG}$ patients before surgery.

\section{PATIENTS AND METHODS}

After the approval of the study protocol by the local ethics committee, informed consent was collected from patients. 


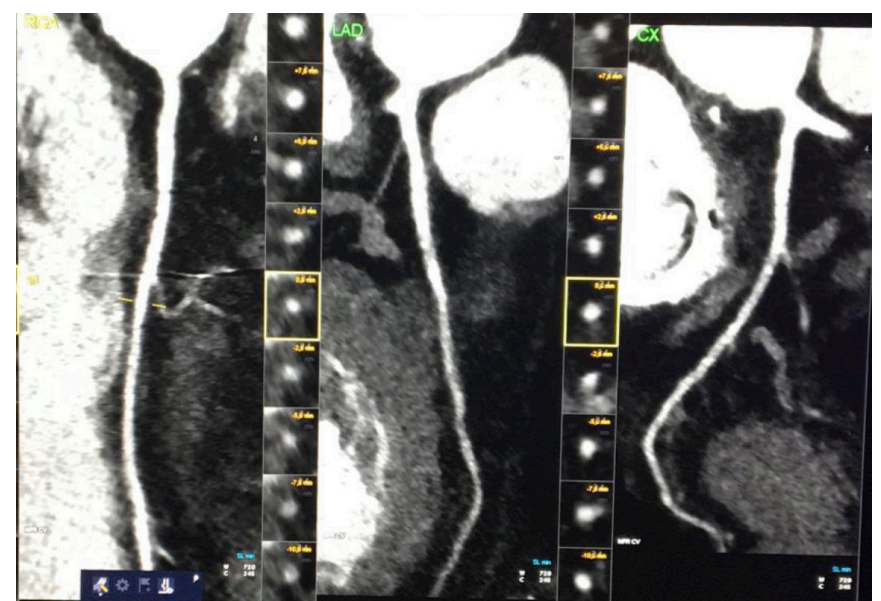

Figure 3. Coronary artery calcification at LAD, CX, and RCA shown in multidetector computed tomography.

The study included a total of 130 consecutive isolated CABG patients, who were treated at our institute, between June 2020 and December 2020. Baseline patients' characteristics are summarized in Table 1 . We excluded patients with cardiac valvular pathology and those who already had a cardiac operation. In addition, patients with a severe peripheral artery disease and those who had low glomerular filtration rate, chronic kidney failure, or hepatic disease also were excluded from the study.

Measurement of CAC and ACS Score: Routine biochemical and blood count values including lipid profiles, preoperative creatinine levels, and estimated glomerular filtration rate (eGFR) were measured before MDCT to detect cardiovascular risk factors. Hypertension was identified based on the prior prescription of antihypertensive drug use. Diabetes was defined as the prior prescription of antidiabetic(s) use. Fasting glucose levels and hemoglobinA1c were measured. Body mass index $(\mathrm{BMI})$ was calculated for each patient.

All MDCTs were performed by an experienced radiologist who was blinded to the study. In order to investigate CAC score and ACS, a non-contrast 128-slice MDCT scanner was used (Somatom Definition, Siemens Healthcare, Forchheim, Germany). CAC scores were calculated, according to the modified Agatston Unit (AU). The scan was performed from the tracheal bifurcation to the diaphragm. Prospective electrocardiogram triggering (75\% of R-R interval) was used with a slice thickness of $2.0 \mathrm{~mm}$. Collimation width and rotation time were $32 \times 0.625 \mathrm{~mm}$ and $330 \mathrm{~ms}$, respectively. All MDCT data were transferred to an off-line workstation (Net Station; Terare Con Inc., San Mateo, CA, USA) for image reconstruction and analysis. ACS and CCS were determined using a 2-D image. For calculation of ascending aortic stiffness, we measured calcific score from the sinotubular junction to the end of the aortic arch (Figures 1 and 2). The tomographic slices were recorded during a single breath-holding session. All images were electronically triggered to minimize cardiac movement. The degree of calcification scores was calculated by multiplying the area of each calcified lesion by a

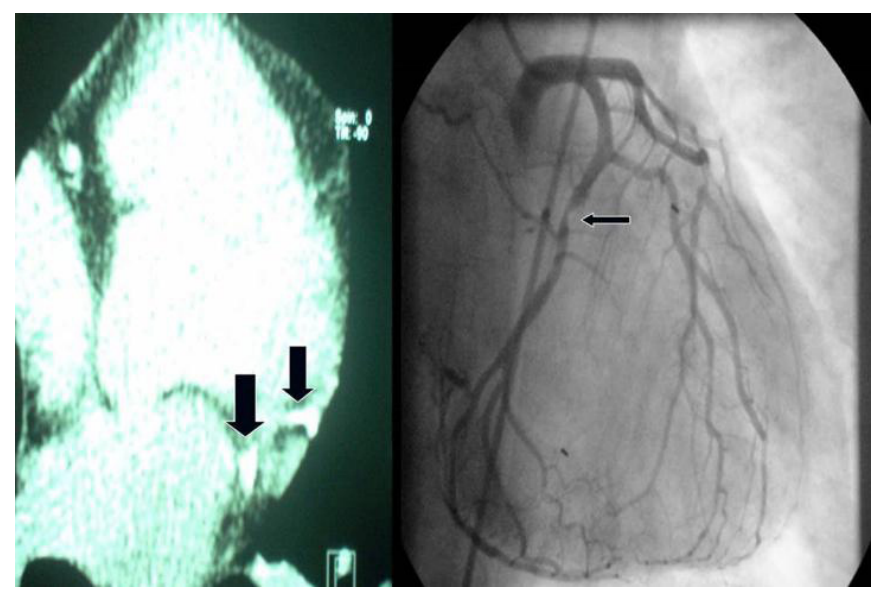

Figure 4. Coronary artery calcification in multidetector computed tomography and conventional angiography.

weighting factor corresponding with the peak pixel intensity for each lesion in order to yield a lesion-specific calcification score. We examined the left main coronary artery, left anterior descending coronary artery (LAD), left circumflex artery $(\mathrm{LCx})$, and the right coronary artery (RCA). According to coronary artery dominancy, 520 coronary segments were evaluated by MDCT in 130 CABG patients, preoperatively. Coronary artery calcification is shown in MDCT following coronary angiography (Figures 3, 4, and 5).

Measurement of SYNTAX score: We performed selective coronary angiography. We determined the presence of a significant lesion if there was a $50 \%$ or greater stenosis in the luminal diameter of the coronary tree. The characteristics of the patients' data, including diseased vessels (LMCA, LAD, RCA, and LCx), and the vessel number were noted. SS is associated with the complexity of diseased coronary arteries, and it was calculated using previously defined software, which integrates some components (morphological features of each coronary artery lesion), including chronic total occlusion (CTO), bifurcation, and trifurcation lesions, tortuosity, and diffuse disease. Lesions with $\geq 50 \%$ luminal obstruction in vessels with a diameter $\geq 1.5 \mathrm{~mm}$ were added to provide www.syntaxscore.com. All coronary angiograms were scored by experienced cardiologists who were unaware of the study.

Pathological evaluation of the aorta: For histologic evaluation, aortic samples were obtained from the lowest concentration of atherosclerotic plaque section that was identified by intraoperative epiaortic ultrasound scanning (EAU). We used a $7.5 \mathrm{MHz}$ ultrasonic probe (Philips EPIQ-7Ultrasound) for evaluation of the ascending aorta intraoperatively before the opening of the pericardium. Concerning the on-pump approach during the cross-clamping or side clamping, a small incision was made on the aorta using No: 11 scalpels. The tissue samples from the ascending aorta were kept in the glutaraldehyde solution under sterile conditions. Tissue samples were processed by routine clinical laboratory methods, being fixed in $10 \%$ formaldehyde and embedded in paraffin wax. Tissue sections were cut, using a microtome, at $5 \mu \mathrm{m}$ thickness, placed onto glass slides, and the sections were stained 


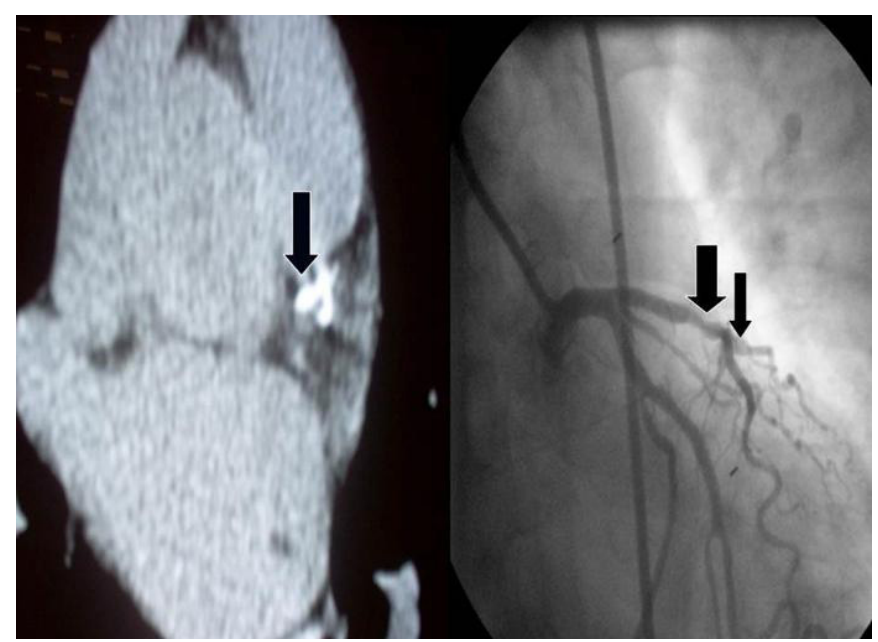

Figure 5. Coronary artery calcification in multidetector computed tomography.

with hematoxylin and eosin (H\&E) and examined under the light microscope. The histochemical evaluation was conducted with in tissue samples, which were classified according to American Heart Association atherosclerosis classification by blinded investigators. The severity of atherosclerosis was classified into six groups compatible with the American Heart Association guidelines. We accepted significant atherosclerosis, in terms of type-3 and greater, which were assigned to the atherosclerosis group (Figure 6). SYNTAX scores for each lesion were calculated separately, and the total SYNTAX scores were obtained by summing all these lesions. According to the ESC guidelines, the SYNTAX score was divided into three groups: less than 15 was considered as low risk (group 1), between 16 and 27 was considered intermediate risk (group 2), and greater than 27 (group 3) was considered as high-risk patients.

Surgical techniques: We used the off-pump technique in patients with isolated LAD stenosis $(\mathrm{N}=18)$. If the patients had no evidence of aortic calcification, then we used a traditional on-pump technique using an aortic cross-clamping $(\mathrm{N}=$ 68 patients) via sternotomy incision. To prevent MACCE and preoperative myocardial ischemia, we used left anterior thoracotomy incision (minimally invasive direct coronary artery bypass) (MIDCAB) in patients with the aortic calcific disease. In patients with severe ACS in combination with multivessel disease and severe aortic calcification or porcelain aorta, arterial and venous cannulation was performed using femoral artery and the right atrium using on-pump techniques.

Patients with a CAC score of less than 400AU, who were operated by the on-pump method without using aortic clamp, were successfully weaned from the extracorporeal circulation. In total arterial CABG, the left and the right internal thoracic artery (LITA and RITA) or the radial artery (RA) were harvested. The T-graft technique was used in patients with ACS $\geq 400(\mathrm{~N}=20)$.

Statistical analysis: All data were processed using the 'Statistical Package for Social Sciences for Windows 22.0 (SPSS, Chicago, IL, USA) program. Continuous variables were

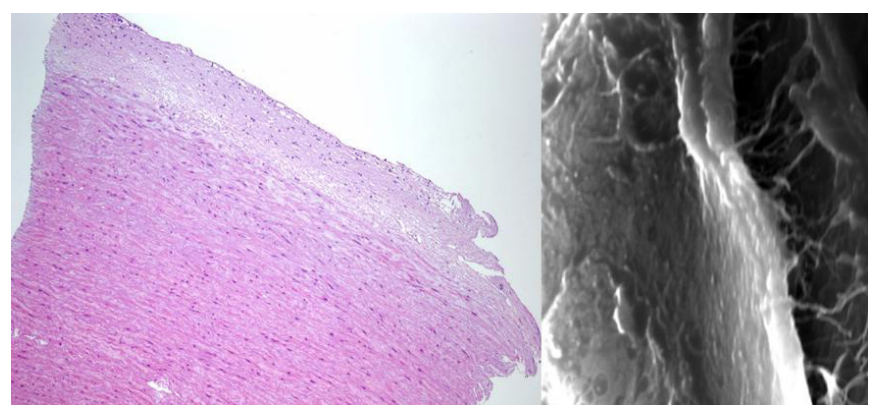

Figure 6. Type 3 and greater atherosclerosis was assigned to significant atherosclerosis group.

expressed as means with standard deviations. Categorical variables were expressed as ratios. The differences between the groups were detected using the $\chi^{2}$-test for categorical variables. Analysis of variance was used for the normally distributed continuous variables, and the Kruskal-Wallis test was utilized for the non-normally distributed continuous variables. Binary logistic regression analysis was performed to test multivariate predictors of atherosclerosis greater than type-3. Receiver operating characteristics (ROC) analysis was used to identify the effects of the SYNTAX I score obtained from coronary angiography lesion assessment on atherosclerotic plaque formation in the ascending aorta. $P$-value <.05 was considered as statistically significant.

Sample size: It was calculated that at least 36 cases should be included in the study when a power analysis was performed with a significance level of 0.05 and a power of 0.80 in order to find a significant relationship with approximately $\mathrm{R}=0.45$ between SYNTAX and CAC scores.

\section{RESULTS}

Four patients died (3\%) during the course of the study. The reasons for death were low cardiac output syndrome (N =3) and acute renal failure $(\mathrm{N}=1)$. We detected a cerebrovascular embolic event in a male patient who had a high ACS (> 1000) $(\mathrm{N}=1)$. Hemiplegia was detected on the third day after surgery in this patient. He was treated and discharged home 34 days after his surgery.

Median SS was $39 \pm 7.2$ (range: $15-41$ ). CAC score and ACS were zero in 34 patients $(26.1 \%)$ and 62 patients $(47.2 \%)$, respectively. The median age of these patients who had no evidence of aortic calcification and CAC were $56 \pm 11.4$ years (range: $37-68$ years) and $59 \pm 14.1$ years (range: $41-67$ years). In these patients, the median SS was $18 \pm 5.4$. Eighteen out of 34 patients had diffuse LAD artery stenosis (13.8\%). Among them, 11 patients had two $(8.4 \%)$, and five patients $(3.8 \%)$ had three vessel diseases, respectively.

The median CAC score was $238 \pm 122$ AU (range: $0-1238$ AU) in 96 CABG patients $(74.9 \%)$, while median ACS was $112 \pm 40$ AU (range: $0-730 \mathrm{AU}$ ) in 68 patients $(52.8 \%)$. Coronary artery calcification was significantly high in patients who had ACS $(P=.002)$ (95\% CI; (0.81-0.93). Sensitivity and specificity were 83.6 and 81.0 , respectively $(P<.001)$. 
Table 1. Demographics and patients

\begin{tabular}{|c|c|c|c|}
\hline \multicolumn{4}{|l|}{ Clinical characteristics } \\
\hline Female gender, n (\%) & $12(17.9)$ & $5(8.2)$ & .106 \\
\hline Body mass index $\left(\mathrm{kg} / \mathrm{m}^{2}\right)$ & $32.87 \pm 3.38$ & $28.20 \pm 4.65$ & .185 \\
\hline Hypertension, n (\%) & $50(74.6)$ & $42(68.9)$ & .468 \\
\hline Hyperlipidemia, n (\%) & $26(38.8)$ & $31(50.8)$ & .172 \\
\hline Smoking, n (\%) & $43(64.2)$ & $39(63.9)$ & .977 \\
\hline Family history, n (\%) & $21(31.3)$ & $31(50.8)$ & .025 \\
\hline Percutaneous coronary intervention, $\mathrm{n}(\%)$ & $18(26.8)$ & $7(11.4)$ & .004 \\
\hline Ejection fraction, \% & $53.10 \pm 7.28$ & $54.18 \pm 8.48$ & .291 \\
\hline SYNTAX score I & $27.38 \pm 8.38$ & $25.31 \pm 6.98$ & .007 \\
\hline EuroSCORE II & $2.93 \pm 1.82$ & $2.10 \pm 2.04$ & .017 \\
\hline \multicolumn{4}{|l|}{ Medication } \\
\hline Aspirin, n (\%) & $32(52.5)$ & $38(56.7)$ & .629 \\
\hline Statins, n (\%) & $24(39.3)$ & $25(37.3)$ & .813 \\
\hline ACE-inhibitors, n (\%) & $25(41)$ & $13(19.4)$ & .008 \\
\hline B blockers, n (\%) & $8(13.1)$ & $7(10.4)$ & .104 \\
\hline
\end{tabular}

The median SYNTAX score was found to be $37.8 \pm 18.3$ for patients whose CAC score was $\geq 400 \mathrm{AU}$. In patients with a CAC score that was lower than $400 \mathrm{AU}$, the median SS was observed to be $19.2 \pm 7.1$. When compared, it was observed that the median SS was significantly higher in patients who had $\geq 400 \mathrm{AU}(P=.000)$. Spearman's correlation test demonstrated a significant correlation between the SS and the total CAC score (R:0.489, $P<.001)$.

In the multivariate analysis of SS (OR 1.053, 95\% CI: $1.003-1.106, P=.039$ ), gender (OR $0.189,95 \%$ CI: $0.053-$ $0.678, P=.011$ ), age (OR 1.454, 95\% CI: $1.256-1.632, P$ $=.012$ ), and diabetes mellitus (OR 0.341, 95\% CI: 1.006$1.124, P=.014$ ) were independent predictors for ACS. ROC curve analysis showed the cut off value of CAC score and ACS in predicting patients with SS $>35$ (Figure 7). Total CAC score identified patients with $\mathrm{SS}>35$ with a specificity of $85 \%(95 \%$ CI: $79.8 \%-90 \%)$ and a sensitivity of $63.2 \%$ (95\% CI:50\%-85\%), and with a cut-off value of > $400 \mathrm{AU}$ (AUC: $0.780,95 \%$ CI: 0.794-0.806). No significant differences were found when compared with CAC and ACS levels in males and females $(64.18 \pm 8.64$ vs. $60.55 \pm 8.28, P=.66)$ $(13.2 \% ; \mathrm{N}=17)$.

Multiple linear regression analysis demonstrated that age ( $\beta: 0.140, P=.024)$, male gender $(\beta: 0.130, P=.032)$, and SS $(\beta: 0.400, P<.000)$ were independent predictors of the total
CAC score. Independent associates of total CAC score are summarized in Table 2. The results of the correlation analysis between the total CAC score and morphological features of lesions included in SS are summarized in Table 3. Pearson's correlation analysis showed that there was a strong correlation between CAC and SYNTAX $(\mathrm{R}=0.366, P=.001)$. None of the independent associates were significantly correlated with the total CAC score $(\mathrm{R}=0.201, P=.002)$. Hypertension, active smoking, preoperative antiaggregant or anticoagulant medication, and dyslipidemia were not significant factors in the SYNTAX level.

The univariate analysis demonstrated that active smoking and dyslipidemia, diabetes, and age were strongly correlated with CAC score levels and aortic stiffness. They were associated with a high CAC score $(P=.01)$. The ANOVA test exhibited significant linearity in the line of regression $(P=$ $.02)$. Age $(\beta=0.208, P=.05)$ and SYNTAX score $(\beta=0.35, P$ $=.004$ ) were found to be significant independent predictors of total CAC score, and aortic calcification. The associations of other variables are shown in Table 4.

The analysis demonstrated that there was a strong relationship between the ACS level (cut-off value $\geq 225 \mathrm{AU}$ ) and SS. In patients who had SS $>35$, the level of CAC was more than $400 \mathrm{AU}(0.88$; $95 \%$ confidence interval, $0.78-0.90$ (sensitivity and specificity were $92.4 \%$ and $78.3 \%(P<.01)$. The 


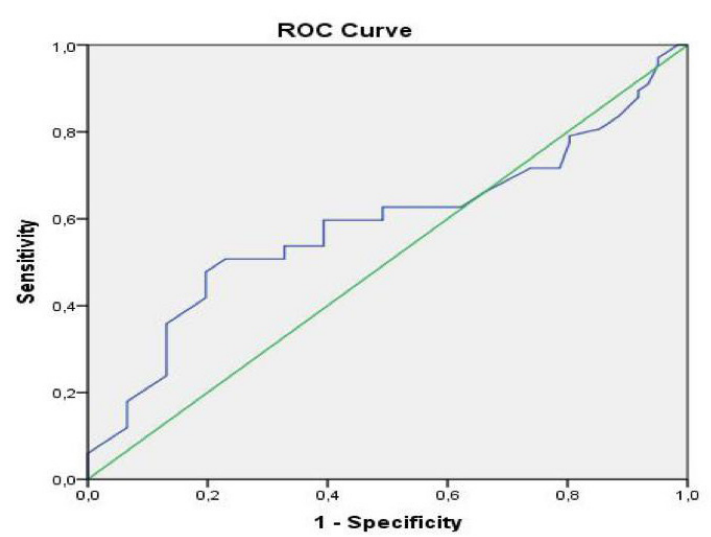

Figure 7. The ROC curve of the relationship between SYNTAX I score and atherosclerosis in aorta.

threshold for predicting a high SYNTAX score was more than $200 \mathrm{AU}(0.81 ; 95 \%$ confidence interval, $0.77-0.87$; sensitivity and specificity were $81.5 \%$ and $90.4 \%$, respectively $(P$ $=.034)$. In addition, the cut-off value of CAC for predicting multivessel disease was $400 \mathrm{AU}(0.70 ; 95 \% \mathrm{CI}, 0.66-0.83)$. The sensitivity and specificity were 84.5 and $72.1 \%$, respectively $(P=.0020)$.

Left ventricular ejection fraction $(\mathrm{LVEF})$ was not related to the CAC score and ACS $(54.18 \pm 8.48$ versus $53.10 \pm$ 7.28; $P=.291)$. The frequency of diabetes mellitus was $29.9 \%(N=20)$ in patients with mild or no atherosclerosis, however in a group of patients with severe atherosclerosis, it was $49.2 \%(\mathrm{~N}=30)$, and the difference was found to be statistically significant $(P=.025)$. Family history was more frequent in patients without or mild atherosclerosis rather than patients with heavy atherosclerosis in the aorta (50.8\% versus $31.3 \%, P=.025)$. As expected, the severe atherosclerosis group had more percutaneous coronary intervention history than the other group, and the difference was statistically significant $(26.8 \%$ versus $11.4 \% ; P=$ .004). Patients with a higher SYNTAX score I tended to have more atherosclerosis than those with lower scores $(27.38 \pm 8.38$ vs. $25.31 \pm 6.98, P=.007)$. EuroScore II was significantly higher in patients with severe atherosclerosis rather than mild atherosclerosis $(2.93 \pm 1.82$ versus $2.10 \pm$ 2.04, $P=.017)$.

Multivariate analysis was performed with adjusting the covariates listed in Table 2. SYNTAX score found an independent predictor [OR 1.053, 95\% CI: 1.003-1.106, $P=.039$ ]. Also, in multivariate analysis, similar results were obtained for gender [OR 0.189, 95\% CI: 0.053-0.678, $P=.011$ ], age [OR 1.454, 95\% CI: $1.256-1.632, P=.012$ ], and DM [OR 0.341, 95\% CI: $1.006-1.124, P=.014]$. SS, gender, advanced age, and DM were found to be independent predictors to predict atherosclerosis in aorta.

The ROC curve represents the relationship between the sensitivity and specificity for the SYNTAX I score to predict atherosclerosis in the aorta (Figure 7). The area under the curve (AUC) between the SYNTAX I score and atherosclerosis in the aorta was $0.585(P=.027)$.
DISCUSSION

Since SS is valuable for the prediction of cardiovascular events in CAD patients, it is being increasingly used to describe the complexity of coronary artery lesions. The relationship between the SYNTAX score and coronary artery calcification in combination with ascending aortic stiffness previously has not been described in CABG patients. A previous study has demonstrated that a higher SYNTAX score $(\geq 20)$ was associated with one-year mortality with a sensitivity of $87.5 \%$ and specificity of $75.7 \%$. Furthermore, the number of coronaries with a CAC score of $>100 \mathrm{AU}$, location of coronary calcification, and several calcific lesions indicated future coronary events [Budoff 2002]. In CABG patients who had a coronary artery calcium score that was greater than 400, the mortality and morbidity risk due to cardiac events, such as preoperative myocardial infarction, was shown to be similar to those patients who had a CAC score below $400 \mathrm{AU}$ in spite of significant CAD in previous studies [Shimizu 2015; Ruben 2018].

We described SYNTAX and CAC score accompanied with aortic stiffness in our $130 \mathrm{CABG}$ patients prior to the surgery and followed up. ACS levels were between 250-1000 AU in 41 of our 71 patients whose CAC score was measured between $250 \mathrm{AU}$ and $1000 \mathrm{AU}$. This result was similar to previously published scientific studies. In these patients, the SYNTAX score value was medium $(\leq 17)$ or severe $\geq 27)$. We observed that the average SYNTAX score value was 27 in our patients whose CAC score and ACS levels were over 1000 AU. We found that we had seven patients with a SYNTAX score greater than or equal to 27.77. We found that our 77 patients with Median CAC score above 100 AU had low SYNTAX values, according to angiography results. In the remaining patients, CAC and ACS were associated with intermediateto-high SYNTAX scores.

Our study showed that there is a positive correlation between the CCS and ACS levels. We also demonstrated that there was a strong correlation between the SYNTAX score as well as the higher CCS with the extension of coronary artery disorder. According to our study, the threshold for detecting a number of diseased vessels is related to the SYNTAX score. In patients with more than $100 \mathrm{AU}$, we observed multivessel disease if CCS is more than 440 AU. Therefore, to prevent MACCE or mortality, we should screen for both ACS and CCS including extending of CAD in patients with a high SYNTAX score before CABG or PCI. If there are calcified plaques in coronary artery trees, generally it needs rotational atherectomy procedures, and surgeons may be aware of any subsequent CAD during the procedure to prevent unexpected hemodynamic instability. According to our results, patients who have scheduled CABG should be screened prior to operations in order to prevent preoperative complications. Since CAC score and ACS measurement are expensive and timeconsuming tests, they may not be used as a standard protocol method in clinical practice. However, according to our study results, we think that in an unexpected MACCE complication that develops after CABG, investigating whether this complication has a high CAC score and/or ACS level 
Table 2. Multivariate analysis of predictors of severe atherosclerosis in the ascending aorta

\begin{tabular}{lccc}
\hline & Significancy & Exp (B) & $95 \% \mathrm{Cl}$ \\
\hline SYNTAX & 0.039 & 1.053 & $1.003-1.106$ \\
EuroSCORE & 0.918 & 0.988 & $0.784-1.244$ \\
Gender* & 0.011 & 0.189 & $0.053-0.678$ \\
Age* & 0.012 & 1.454 & $1.256-1.632$ \\
Diabetes mellitus* & 0.014 & 1.045 & $1.006-1.124$ \\
Nagelkerke R Square & 0.596 & &
\end{tabular}

can be scientifically used to explain the complication. Thus, according to our results, the cardiac team may decide to perform MDCT preoperatively or prior to PCI in older CABG patients with a high SS.

Calcium deposition in all vascular trees, including the aortic wall and coronary arteries, may be seen as a reflection of systemic atherosclerosis. Therefore, quantitative ACS and CAC assessment after SYNTAX score measurement would be more valuable information for evaluating the degree of CAD using MDCT. Our results can encourage clinicians to investigate not only ACS, but also CAC scores in CABG patients, even though quantitative calcium scoring is difficult to use with MDCT.

The correlation between classical cardiovascular risk factors and the development of atherosclerosis has been well established in several trials. However, the correlation between the severity of atherosclerosis and traditional risk scoring systems was not well established. Various studies demonstrated the relationship between the risk scoring systems and peripheral vascular diseases, but there have not been any studies to investigate the correlation between the severity of atherosclerosis in the aortic specimen and coronary artery risk scoring systems.

The principal findings of our study are that the scores demonstrating coronary artery complexity correlate with the severity of atherosclerosis of the ascending aorta. SYNTAX I and EuroSCORE II were found to be significantly higher in the group with atherosclerosis in the ascending aorta by univariate analyses. SYNTAX I and EuroSCORE II were shown as independent predictors of severe atherosclerosis in the ascending aorta in multivariate analyses performed to get rid of the effects of other causes that may cause atherosclerosis, such as age and gender.

SYNTAX score was advocated in cardiology guidelines to define the extensiveness of coronary artery disease and accordingly decide the optimum revascularization type. Although atherosclerosis is a systematic disease, the direct association between obstructive coronary artery disease and non-coronary atherosclerosis is uncertain. Contemporary studies demonstrate the correlation between non-coronary atherosclerosis and obstructive coronary disease. Furthermore, systematic atherosclerosis is likely to increase the risk of myocardial infarction, stroke, and all kinds of mortality [Ruben 2018; Mirbolouk 2020].

Some studies have been conducted in the past to show that scoring systems, such as SYNTAX which determine the severity of coronary artery diseases, can predict diseases occurring in other arterial systems other than coronary arteries. Abdelnabi et al reported that SYNTAX II score and carotid intima-media thickness were strongly correlated in their series of 155 patients [Abdelnabi 2020]. They showed that the median SYNTAX II score and mean intima-media thickness of carotid arteries were $10.23 \pm 11.36$ and $0.85 \pm$ 0.24, respectively [Abdelnabi 2020].

Atherosclerosis mainly is seen as a result of imbalanced lipid metabolism and inconvenient chronic inflammatory response of the vascular endothelium. The association of plasma lipid parameters and severity of atherosclerosis in ascending aorta was demonstrated previously by obtaining aortic tissue specimens on the aortic wall. According to a study, the extension of atherosclerosis is positively correlated with plasma TC, LDL-C, and TG concentrations, and negatively correlated with HDL-C concentration [Shabbir 2021].

A study, in which the thoracic aortic atherosclerotic plaques was diagnosed by transesophageal echocardiography, reported atherosclerotic plaque formation in the thoracic aorta that was $90 \%$ sensitive with $90 \%$ specificity to predict coronary artery disease [Shabbir 2021]. However, the association between ascending aorta, aortic arch, and coronary arteries was not found to be significant. The association between obstructive coronary artery disease and atherosclerotic plaque previously has been reported in several studies by using transesophageal echocardiography, ultrasonography, and magnetic resonance imaging [Berman 2016; Malguria 2018]. Cardiac magnetic resonance imaging also can be routinely used; however, it is not feasible because of the high costs. $\mathrm{Li}$ et al $[\mathrm{Li}$ 2016] classify atherosclerotic plaques by severity and extensivity of disease. In multivariate analysis, abdominal aortic plaques were independent predictors associated with the presence of obstructive coronary artery disease (OR: 2.08; 95\% Cl: 1.50-2.90; $P<.001)$.

Recent studies stated the extent of atheromatous plaque burden in the aorta was an independent risk factor in cardiothoracic surgery. Heavy plaque formation in the thoracic, ascending aorta, and aortic arch was related to increased cerebrovascular events and mortality. Kurra et al [Kurra 2010] reported that the enhanced plaque burden score was related to long-term mortality in patients after cardiothoracic surgery (HR: $1.08 ; P<.001)$. The pathological mechanism has not been clearly understood yet; however, particular manipulations of the aorta during surgery, perioperative plaque 
Table 3. Data of multislice computed tomography

\begin{tabular}{lcc}
\hline ACS score (AU) (mean, range) & $123(24-440)$ & \\
CAC score (AU) (mean, range) & $401(54-980)$ & \\
Number of coronary artery disease & Median CAC Score & \\
Single vessel (n) & 18 & 0 \\
Two vessel ( $n)$ & 11 & 349 \\
Three vessel (n) & 85 & 586 \\
$\geq 4$ vessel disease $(n)$ & 16 & 790 \\
\hline
\end{tabular}

ACS, aortic calcific score; CCS, coronary calcium score; AU, Agatston unit

embolization, and cerebrovascular events were accused. Although the SYNTAX score is an important prognostic parameter for PCI, a significant effect on survival in bypass operation has not been demonstrated by recent studies. SYNTAX Trial has shown that PCI applications in patients with high SYNTAX scores reduce the risk of stroke seen in CABG surgeries. For this reason, it has been suggested by the authors that PCI should be preferred in patients with high SYNTAX value in order to reduce strokes during revascularization [Kurra 2010]. Gokdeniz et al showed in symptomatic patients with accompanying significant CAD, total CAC score was independently associated with SYNTAX score. Patients with SS $>32$ have high arterial calcific disorders [Gokdeniz 2014]. The findings of this study were similar to our results.

According to our study results, DM is an independent predictor of atherosclerosis in the ascending aorta and correlated with SYNTAX score. DM refers to a hyperglycemic situation that is induced by oxidative stress, inflammation, and pro-inflammatory responses to reactive oxygen species [Vroegindewey 2018]. The pathogenesis of both atherosclerosis and DM are similar; however, the interaction between them is not yet certain. Advanced age and male gender are well-known risk factors for cardiovascular diseases in our study. Both were observed among the risk factors that increase the severity of atherosclerosis in the ascending aorta.

Prediction of the plaque structure in the aorta is vitally important because of the risk assessment and prevention of complications in operations, such as CABG, where the ascending aorta is manipulated and clamped. Calcified aorta, prior history of cerebrovascular disease, advanced age, carotid arterial disease, duration of cardiopulmonary bypass, renal failure, cigarette smoking, and diabetes mellitus were the risk factors for postoperative stroke in a study, which included 20,000 patients who underwent CABG [Farooq 2013; Gutierrez 2019].

Haberal et al showed that no significant differences were found between HDL-C, LDL-C, triglyceride, total cholesterol values, and the pathological process of aortic atherosclerosis [Haberal 2020]. They suggested another investigation of biochemical parameters in these particular patients to explain other risk factors of aortic stiffness [Haberal 2020]. Gu et al demonstrated using multivariate analysis that included hypertension, diabetes mellitus, and aortic plaque were significantly associated with CAD [Gu 2011]. A study by Kronzon et al
Table 4. Association of total Aortic calcification score in multivariate linear regression analysis

\begin{tabular}{lcc}
\hline & Variables Standardized Coefficients & $P$ \\
\hline Age* (years) & 0.140 & .024 \\
No. of CAD & -0.055 & .340 \\
Hemoglobin, g/dL & -0.060 & .245 \\
LVEF (\%) & -0.000 & .890 \\
SYNTAX* & 0.400 & $<.000$ \\
Male* & 0.130 & .032 \\
HT & 0.044 & .476 \\
DM* & 0.041 & .560 \\
\hline
\end{tabular}

CAD, coronary artery disease; LVEF, left ventricular ejection fraction; HT, hypertension; DM, diabetes mellitus

demonstrated the relationship between severity of aortic atherosclerosis and stroke rate. According to this research, an intense aortic plaque, especially in the ascending and aortic arch, might be the cause embolic events [Kronzon 2006]. In another publication, Amarenco et al proposed that atherosclerotic changes of carotid artery systems were also an important cause of stroke. In this study, Amarenco et colleagues showed that plaque size in the ascending aorta of more than $4 \mathrm{~mm}$ is an independent predictor of ischemic stroke preoperatively [Amarenco 1994]. Avoidance of aortic manipulation during off-pump CABG decreases embolic events compared with cardiac surgery technique [Misfeld 2011]. According to our experiences, we recommend off-pump coronary artery bypass grafting surgery with the use arterial bypass graft in patients who have a history of ischemic attack. Ascending aortic calcification significantly enhanced postoperative stroke risk.

Avoiding undesirable cerebrovascular accidents during surgery is crucial. Minimally invasive techniques, avoidance of extreme aortic manipulation, and prolonged ECC time should be considered in case of high ascending aortic stiffness due to plaque load [Misfeld 2011]. In previous studies, the authors exhibited that stroke rates decreased by $70 \%$ in CABG "without touching the aorta" in risky patients with high SYNTAX value [Gutierrez 2019; Haberal 2020; Gu 2011; Kronzon 2006; Amarenco 1994]. For this reason, we performed the surgeries based on the SYNTAX values for each patient separately before CABG operations, and we did not observe any unexpected cerebrovascular accident.

Study limitations: The main limitation of our study is that its duration was one year. Our study has a limited number of patients and relatively short follow-up period. Therefore, we need a large case series for the interpretation of variables.

\section{CONCLUSION}

Aortic and coronary artery atherosclerotic plaque are the main factors for stroke, preoperative myocardial ischemia, 
and infarction in CABG patients. According to our study results, CAC score and aortic stiffness have correlated with SS in elderly CABG patients, who had diabetes mellitus. We believe that elderly CABG patients with high SS and CAC scores should be followed up more closely. Despite the small number of patients, an important finding of our study results is that the SYNTAX score value and aortic atherosclerosis levels are directly related to each other. Accordingly, in patients who will undergo elective CABG (especially in octogenarian patients), in the case of high SYNTAX values in the preoperative period, the measurement of aortic calcification values by MDCT and planning the CABG technique according to the patient may positively affect mortality and morbidity.

\section{REFERENCES}

Abdelnabi M, Almaghraby A, Tok OO, Oz TK, et al. 2020. A real-life correlation between clinical SYNTAX score II and carotid intima-media thickness in patients with stable coronary artery disease. J Saudi Heart Assoc. 32:8-11.

Amarenco P, Cohen A, Tzourio C, Bertrand B, et al. 1994. Atherosclerotic disease of the aortic arch and the risk of ischemic stroke. $\mathrm{N}$ Engl J Med. 331:1474-1479.

Berman DS, Arnson Y, Rozanski A. 2016. Coronary artery calcium scanning: the Agatston score and beyond. JACC Cardiovasc Imaging. 9:1417-9.

Budoff MJ, Diamond GA, Raggi P, Arad Y, et al. 2002. Continuous probabilistic prediction of angiographically significant coronary artery disease using electron beam tomography. Circulation. 105:1791-6.

Farooq V, Serruys PW, Bourantas CV, Zhang Y, Muramatsu T, Feldman T, et al. 2013. Quantification of incomplete revascularization and its association with five-year mortality in the synergy between percutaneous coronary intervention with taxus and cardiac surgery (SYNTAX) trial validation of the residual SYNTAX score. Circulation. 128 (2):141-51.

Gokdeniz T, Kalaycioglu E, Aykan AC, Boyaci F, et al. 2014. Value of coronary artery calcium score to predict severity or complexity of coronary artery disease. Arq Bras Cardiol. 102:120-7.

Gu X, He Y, Li Z, Kontos MC, et al. 2011. Relation between the incidence, location, and extent of thoracic aortic atherosclerosis detected by transesophageal echocardiography and the extent of coronary artery disease by angiography. Am J Cardiol. 107:175-8.

Gutierrez JA, Bhatt DL, Banerjee S, Glorioso TJ, et al. 2019. Risk of obstructive coronary artery disease and major adverse cardiac events in patients with noncoronary atherosclerosis: Insights from the Veterans Affairs Clinical Assessment, Reporting, and Tracking (CART) Program. Am Heart J. 213:47-56.

Haberal I, Yesiltas MA, Koyuncu AO, Batur S, et al. 2020. Is it possible to predict atherosclerosis in the ascending aorta by the patient's lipid panel Arch Med Sci Atheroscler Dis. 5:237-244.

Haberl R, Becker A, Leber A, Knez A, et al. 2001. Correlation of coronary calcification and angiographically documented stenoses in patients with suspected coronary artery disease: results of 1,764 patients. J Am Coll Cardio. 37:451-7.

Herrington W, Lacey B, Sherliker P, Armitage J, Lewington S. 2016. Epidemiology of Atherosclerosis and the Potential to Reduce the Global
Burden of Atherothrombotic Disease. Circ Res. 118(4):535-46.

Kojima K, Kimura S, Hayasaka K, Mizusawa M, et al. 2019. Aortic Plaque Distribution, and Association between Aortic Plaque and Atherosclerotic Risk Factors: An Aortic Angioscopy Study. J Atheroscler Thromb. 1;26(11):997-1006.

Kronzon I, Tunick PA. 2006. Aortic Atherosclerotic Disease and Stroke. Circulation. 114:63-75.

Kurra V, Lieber ML, Sola S, Kalahasti V, et al. 2010. Extent of thoracic aortic atheroma burden and long-term mortality after cardiothoracic surgery: a computed tomography study. JACC Cardiovasc Imaging. $3: 1020-9$.

Li W, Luo S, Luo J, Liu Y, et al. 2016. Association between abdominal aortic plaque and coronary artery disease. Clin Interv Aging. 11:683-8.

Malguria N, Zimmerman S, Fishman EK. 2018. Coronary artery calcium scoring: current status and review of literature. J Comput Assist Tomogr. 42:887-97.

Mirbolouk M, Kianoush S, Dardari Z, Miedemad MD, et al. 2020. The association of coronary artery calcium score and mortality risk among smokers: The coronary artery calcium consortium. Atherosclerosis. 294:33-40.

Misfeld M, Brereton RJ, Sweetman EA, Doig GS. 2011. Neurologic complications after off-pump coronary artery bypass grafting with and without aortic manipulation: meta-analysis of 11,398 cases from 8 studies. J Thorac Cardiovasc Surg. 142:11-7.

Nohara H, Shida T, Mukohara N, Obo H, et al. 2004. Ultrasonic plaque density of aortic atheroma and stroke in patients undergoing on-pump coronary bypass surgery. Ann Thorac Cardiovasc Surg. 10(4):235-40.

Ozturk D, Celik O, Erturk M, Kalkan AK, et al. 2016. Utility of the Logistic Clinical Syntax Score in the Prediction of Contrast-Induced Nephropathy After Primary Percutaneous Coronary Intervention. Can J Cardiol. 32(2):240-6.

Raggi P, Callister TQ, Cooil B, He ZX, et al. 2000. Identification of patients at increased risk of first unheralded acute myocardial infarction by electron-beam computed tomography. Circulation. 101:850-5.

Rencuzogullari I, Çağdaş M, Karakoyun S, Yesin M, et al. 2018. Propensity score matching analysis of the impact of Syntax score and Syntax score II on new onset atrial fibrillation development in patients with ST segment elevation myocardial infarction. Ann Noninvasive Electrocardiol. 23:12504.

Ruben VC, Buijs EL, Leemans MG, Ignace FJ, et al. 2018. Quantification of abdominal aortic calcification: Inherent measurement errors in current computed tomography imaging. PLoS One. 13: e0193419.

Schaftenaar F, Frodermann V, Kuiper J, Lutgens E. 2016. Atherosclerosis: the interplay between lipids and immune cells. Curr Opin Lipidol. 27(3):209-15.

Shabbir A, Virk ST, Malik J, Kausar S, et al. 2021. Coronary Artery Calcium Score: Assessment of SYNTAX Score and Prediction of Coronary Artery Disease. Cureus. 13(1):12704.

Shimizu K, Yamamoto M, Koyama Y, Kodama A, et al. 2015. Usefulness of routine aortic valve calcium score measurement for risk stratification of aortic stenosis and coronary artery disease in patients scheduled cardiac multislice computed tomography. Int J Cardiol Heart Vasc. 7; 9:95-99.

Vroegindewey MM, Schuurman AS, Oemrawsingh RM, van Geuns RJ, et al. 2018. SYNTAX score II predicts long-term mortality in patients with one- or two-vessel disease. PLoS One. 2:13:e0200076. 